\title{
Quadrotor Angle Stabilization using Full State Feedback by Partial Robust Pole Assignment Method: Pole Retention
}

\author{
Mustafa Saraoğlu \\ Institute of Automation \\ Technische Universität Dresden \\ Dresden, Germany \\ mustafa.saraoglu@tu-dresden.de \\ Andrey Morozov \\ Institute of Automation \\ Technische Universität Dresden \\ Dresden, Germany \\ andrey.morozov@tu-dresden.de
}

\author{
Klaus Janschek \\ Institute of Automation \\ Technische Universität Dresden \\ Dresden, Germany \\ klaus.janschek@tu-dresden.de \\ Mehmet Turan Söylemez \\ Control and Automation Engineering Department \\ Istanbul Technical University \\ Istanbul, Turkey \\ turan.soylemez@itu.edu.tr
}

\begin{abstract}
Quadrotor also referred to as quadcopter has an increasing reputation nowadays as a practical rotorcraft and an unmanned aerial vehicle (UAV). The high number of degrees of freedom makes stability a serious issue as well as a performance criterion. Although, many different methods and strategies of control are applied to different models of quadrotors, an important issue that has not been covered widely is the robustness of the control and parametric uncertainty notions. Robust control seeks to design systems which work despite changing operating conditions, measurement errors and unknown affects. This paper presents a deep analysis of the robust pole assignment method for full state feedback-controlled quadrotor. A new numerical method for a multi-linear coefficient uncertain polynomial is introduced using quasi-gamma stability. Pole retention method is used as a robust pole assignment method for designing the feedback controller to guarantee the stability of the system for an interval of state feedback coefficients. The initial state response of the uncertain quadrotor system is shown to verify the system's performance.
\end{abstract}

Keywords-Unmanned aerial vehicles, state feedback, pole assignment, robust control

\section{INTRODUCTION}

Quadrotor or sometimes referred to as quadcopter is an unmanned aerial vehicle, which can be controlled by remote controllers. Quadrotor consists of four rotors at each end of its X-shaped chassis. These four rotors enable quadrotors to climb, hover, pitch, yaw, roll and also move in lateral directions.

Quadrotors may be used in various ways, such as for surveillance with added vision elements. Proving useful in such operations such as detection, search, and rescue, interest in quadrotors have increased. Being relatively small, agile and artificially intelligent thanks to the developing technologies of microcontrollers, quadrotors will become more popular in the coming years [1].

The first known type of quadrotor in the literature is known as Bréguet Richet Gyroplane constructed by two brothers Louis Bréguet and Jacques Bréguet in 1907. In 1922, Georges de Bothezat and Ivan Jerome constructed a six-rotor vehicle with a similar structure to the nowadays quadrotor [2]. A lot of different designs and perspectives are present in the past until the construction of helicopters and quadrotors. Vertical take-off and hovering ability of implemented rotors are the key structures which differ them from most of the air-planes.

Quadrotors capabilities of motion involve few differences from the conventional aircraft. For instance, most aircraft need longitudinal speeds to change their altitudes since they do not have rotors that can lift the whole aircraft vertically. However, quadrotors have the advantage of four balanced rotors [3]. To achieve stability and to control the quadrotors, four rotors turn at independent speeds from each other. To lift the rotor vertically without any angular disposition, four rotors can be operated at the same speed to create an upward thrust. Control problem of a quadrotor is examined in various published papers concerning different state variables for different motions and rotations in [4]. Analysis of the motions of quadrotors will be examined in the next section.

Another key feature to be presented in this paper is the notion of partial pole assignment and the robustness analysis for assigned poles. A model quadrotor will be examined concerning its angle stability with state feedback and possible feedback gain coefficients regarding some desired criteria. To solve the determination of the feedback gain coefficients as multi-linear coefficients, a new numerical approach concerning gamma-stability will be introduced [5]. Then the performance of the assigned poles will be simulated for the possible range of stabilizing feedback gain values that are found by the introduced method.

\section{QUADROTOR EQUATIONS OF MOTIONS}

\section{A. Rotational Equations of Motions}

Rotational motions are similarly obtained by the thrust of four rotors located on the quadrotor. Rotors turn at different velocities to create a difference between thrust forces which eventually turns the quadrotor around its main axes. This way, three different rotational motions are achieved. To keep the altitude same or raise, all rotors have to turn. So while keeping the altitude same, rotational motions are achieved by relatively faster or slower angular speeds for different rotors. 
Main angular directions are needed to be introduced for notational clearance. Three angular motions are denoted by roll angle $(\phi)$, pitch angle $(\theta)$ and yaw angle $(\psi)$.

Fig. 1 below demonstrates how the rotational motions and vertical motion is achieved.

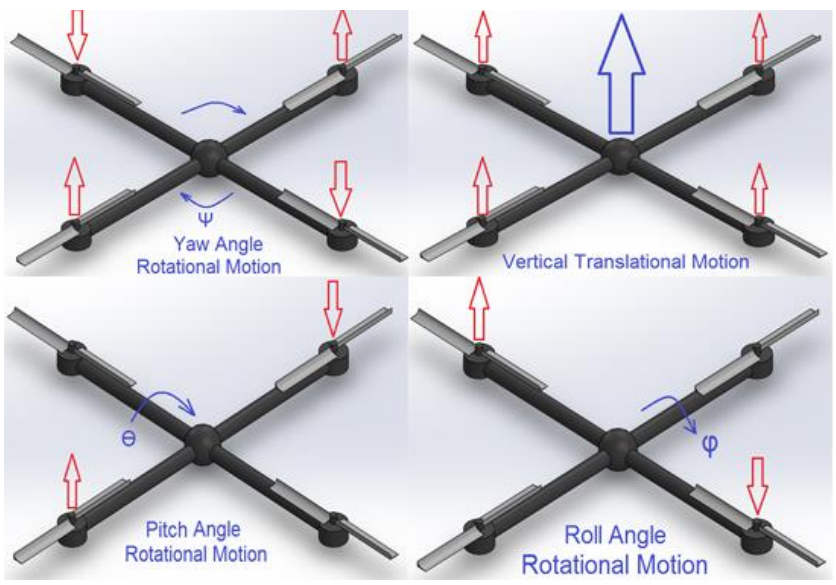

Fig. 1. Rotational and vertical motions: How thrust forces created by rotors affect motions.

The motions of equations can be extended to cover all translational and rotational motions but the focus of the paper is about rotational motions and for the quadrotor, rotational motions and the translational motions can be decoupled without a problem around an operating point.

The equations of motions are given below in terms of states and inputs. Normally, the input can be expanded into the relationship between the command signal voltage and the thrust created by the rotors. However, this would just make further complications. We can represent the torque that is created solely as inputs and this approach would just work fine in order to design a state feedback controller. From Newton's Second Law for rotational motions (1) can be derived:

$$
\begin{aligned}
& I_{x x} \ddot{\Phi}=\dot{\Theta} \dot{\Psi}\left(I_{y y}-I_{z z}\right)+J_{r} \dot{\Theta} \Omega_{r}+u_{1} \\
& I_{y y} \ddot{\Theta}=\dot{\Phi} \dot{\Psi}\left(I_{z z}-I_{x x}\right)+J_{r} \dot{\Phi} \Omega_{r}+u_{2} \\
& I_{z z} \ddot{\Psi}=\dot{\Theta} \dot{\Phi}\left(I_{x x}-I_{y y}\right)+u_{3}
\end{aligned}
$$

To represent the system in terms of states and inputs, a state vector is introduced consisting of three angles and their rates of change. The rotational equations of motions are as follows in (2), (3), (4) and the constants in (2) are given in (5):

$$
f(x, u)=\left\{\begin{array}{c}
x_{2} \\
a_{1} x_{4} x_{6}+a_{2} \Omega_{r} x_{4}+b_{1} u_{1} \\
x_{4} \\
a_{3} x_{2} x_{6}+a_{4} \Omega_{r} x_{2}+b_{2} u_{2} \\
x_{6} \\
a_{5} x_{4} x_{2}+b_{3} u_{3}
\end{array}\right\}
$$

$$
\begin{gathered}
f(x, u)=\left|\begin{array}{c}
\Phi \\
\dot{\Phi} \\
\Theta \\
\dot{\Theta} \\
\Psi \\
\dot{\Psi}
\end{array}\right|=\left|\begin{array}{c}
x_{1} \\
x_{2} \\
x_{3} \\
x_{4} \\
x_{5} \\
x_{6}
\end{array}\right| \\
\dot{x}=f(x, u)
\end{gathered}
$$

\section{B. Moment of Intertia and Other Constants}

The constants regarding the structure and the nature of

\begin{tabular}{|c|c|c|c|}
\hline Constants & Values & Units & Description \\
\hline $\mathrm{I}_{\mathrm{xx}}, \mathrm{I}_{\mathrm{yy}}$ & $6.12233 \mathrm{e}-005$ & $\mathrm{Kg} \mathrm{m}^{2}$ & $\begin{array}{c}\text { Moment of inertia along } \\
\qquad \phi \text { and } \theta\end{array}$ \\
\hline $\mathrm{I}_{\mathrm{zz}}$ & $8.11858 \mathrm{e}-005$ & $\mathrm{Kg} \mathrm{m}^{2}$ & $\begin{array}{c}\text { Moment of inertia along } \\
\psi\end{array}$ \\
\hline $\mathrm{M}$ & 0.5 & $\mathrm{Kg}$ & Quadrotor mass \\
\hline 1 & 0.235 & $\mathrm{~m}$ & $\begin{array}{l}\text { Distance between center } \\
\text { of mass and the rotor }\end{array}$ \\
\hline$\Omega_{\mathrm{r}}$ & 0.125 & $\operatorname{Rad} / \mathrm{s}$ & $\begin{array}{c}\text { Overall residual propeller } \\
\text { angular speed }\end{array}$ \\
\hline $\mathrm{J}_{\mathrm{r}}$ & $6 e-5$ & $\mathrm{Kg} \mathrm{m}^{2}$ & Rotor's moment of inertia \\
\hline
\end{tabular}
the problem are given in Table 1. Values for the moments of inertia and the mass are approximated as in the work [4]. Other variables seen in (2) are shortened versions which can be determined from (5).

TABLE I. THE CONSTANTS AND DESCRIPTIONS

$$
\begin{aligned}
& a_{1}=\frac{I_{y y}-I_{z z}}{I_{x x}}, a_{2}=\frac{J_{r}}{I_{x x}}, a_{3}=\frac{I_{z z}-I_{x x}}{I_{y y}}, \\
& a_{4}=\frac{J_{r}}{I_{y y}}, a_{5}=\frac{I_{x x}-I_{y y}}{I_{z z}}, \\
& b_{1}=\frac{I}{I_{x x}}, b_{2}=\frac{I}{I_{y y}}, b_{3}=\frac{I}{I_{z z}}
\end{aligned}
$$

\section{State Space MOdel OF THE QuAdrotor}

\section{A. Modeling the Open-loop Dynamics of the System}

As we derived the equations of motions for the quadrotor, we can simply express the governing motions in state-space format. Firstly, the system must be linear so a linearization is necessary around the operating point concerning small angle assumption. Multiplications of the state variables for small angles and the gyro effects due to overall residual propeller angular speed are neglected. Remaining A and B matrices after linearization can be seen from (6), (7) and (8):

$$
\dot{x}=A x+B u
$$




$$
\begin{aligned}
A & =\left|\begin{array}{llllll}
0 & 1 & 0 & 0 & 0 & 0 \\
0 & 0 & 0 & 0 & 0 & 0 \\
0 & 0 & 0 & 1 & 0 & 0 \\
0 & 0 & 0 & 0 & 0 & 0 \\
0 & 0 & 0 & 0 & 0 & 1 \\
0 & 0 & 0 & 0 & 0 & 0
\end{array}\right| \\
B & =\left|\begin{array}{lll}
0 & 0 & 0 \\
b_{1} & 0 & 0 \\
0 & 0 & 0 \\
0 & b_{2} & 0 \\
0 & 0 & 0 \\
0 & 0 & b_{3}
\end{array}\right|
\end{aligned}
$$

\section{B. Modelling the State Feedback Closed-loop Dynamics of} the System

State Feedback approach is necessary to stabilize the system. Each state can be stabilized, if its rate of change is taken into consideration. Otherwise, just controlling the angles can only place the poles of the closed-loop system on the imaginary axis causing the system to be marginally stable at best. Therefore, the state feedback matrix should consist of 3 rows and 6 columns. It is known that the motions of the quadrotor can be decoupled from each other so the following matrix can be used as a state feedback matrix:

$$
K=\left|\begin{array}{cccccc}
k_{1} & k_{2} & 0 & 0 & 0 & 0 \\
0 & 0 & k_{3} & k_{4} & 0 & 0 \\
0 & 0 & 0 & 0 & k_{5} & k_{6}
\end{array}\right|
$$

The state feedback law shown in (10) is applied to the system:

$$
u=-K x
$$

The characteristic equation is found [6] as follows (11):

$$
\begin{aligned}
& \dot{x}=A x+B u \\
& u=-K x \\
& \dot{x}=x(A-B K) \\
& A_{c l}=A-B K \\
& P(s)_{c h}=\operatorname{det}\left(s I-A_{c l}\right) .
\end{aligned}
$$

Using (7), (8) and (9) in (11) the following expression is obtained for the closed-loop system characteristic equation (12):

$$
\begin{aligned}
& P(s)_{c h}=s^{6}+s^{5}\left(b_{1} k_{2}+b_{2} k_{4}+b_{3} k_{6}\right)+s^{4} \\
& \left(b_{1} k_{1}+b_{2} k_{3}+b_{1} b_{2} k_{2} k_{4}+b_{3} k_{5}+b_{1} b_{3} k_{2} k_{6}\right. \\
& \left.+b_{2} b_{3} k_{4} k_{6}\right)+s^{3}\left(b_{1} b_{2} k_{2} k_{3}+b_{1} b_{2} k_{1} k_{4}+\right. \\
& b_{1} b_{3} k_{2} k_{5}+b_{2} b_{3} k_{4} k_{5}+b_{1} b_{3} k_{1} k_{6}+b_{2} b_{3} k_{3} k_{6} \\
& \left.+b_{1} b_{2} b_{3} k_{2} k_{4} k_{6}\right)+s^{2}\left(b_{1} b_{2} k_{1} k_{3}+b_{1} b_{3} k_{1} k_{5}\right. \\
& +b_{2} b_{3} k_{3} k_{5}+b_{1} b_{2} b_{3} k_{2} k_{4} k_{5}+b_{1} b_{2} b_{3} k_{2} k_{3} k_{6} \\
& \left.+b_{1} b_{2} b_{3} k_{1} k_{4} k_{6}\right)+s\left(b_{1} b_{2} b_{3} k_{2} k_{3} k_{5}+\right. \\
& \left.b_{1} b_{2} b_{3} k_{1} k_{4} k_{5}+b_{1} b_{2} b_{3} k_{1} k_{3} k_{6}\right)+\left(b_{1} b_{2} b_{3} k_{1} k_{3} k_{5}\right) .
\end{aligned}
$$

\section{Angle Stabilization With State FeEdBack}

\section{A. Kharitonov Polynomials Approach}

The closed-loop characteristic polynomial depends on 6 free parameters of the controller $(\mathrm{k} 1 . . \mathrm{k} 6)$, as well as the parameters of the B matrix. An overbounding polynomial can be constructed in order to change the problem of multilinear coefficients into interval polynomials and to be able to use Kharitonov's Theorem [7]:

$P_{o p}=s^{6}+s^{5} \alpha_{5}+s^{4} \alpha_{4}+s^{3} \alpha_{3}+s^{2} \alpha_{2}+s \alpha_{1}+\alpha_{0}$

$\alpha_{5}=b_{1} k_{2}+b_{2} k_{4}+b_{3} k_{6}$,

$\alpha_{4}=b_{1} k_{1}+b_{2} k_{3}+b_{1} b_{2} k_{2} k_{4}+b_{3} k_{5}+b_{1} b_{3} k_{2} k_{6}$

$+b_{2} b_{3} k_{4} k_{6}$,

$\alpha_{3}=b_{1} b_{2} k_{2} k_{3}+b_{1} b_{2} k_{1} k_{4}+$

$b_{1} b_{3} k_{2} k_{5}+b_{2} b_{3} k_{4} k_{5}+b_{1} b_{3} k_{1} k_{6}+b_{2} b_{3} k_{3} k_{6}$

$+b_{1} b_{2} b_{3} k_{2} k_{4} k_{6}$

$\alpha_{2}=b_{1} b_{2} k_{1} k_{3}+b_{1} b_{3} k_{1} k_{5}$

$+b_{2} b_{3} k_{3} k_{5}+b_{1} b_{2} b_{3} k_{2} k_{4} k_{5}+b_{1} b_{2} b_{3} k_{2} k_{3} k_{6}$

$+b_{1} b_{2} b_{3} k_{1} k_{4} k_{6}$,

$\alpha_{1}=b_{1} b_{2} b_{3} k_{2} k_{3} k_{5}+b_{1} b_{2} b_{3} k_{1} k_{4} k_{5}+b_{1} b_{2} b_{3} k_{1} k_{3} k_{6}$,

$\alpha_{0}=b_{1} b_{2} b_{3} k_{1} k_{3} k_{5}$.

$P_{o p}^{--}=s^{6}+s^{5} \alpha_{5}^{-}+s^{4} \alpha_{4}^{-}+s^{3} \alpha_{3}^{+}+s^{2} \alpha_{2}^{+}+s \alpha_{1}^{-}+\alpha_{0}^{-}$

$P_{o p}^{-+}=s^{6}+s^{5} \alpha_{5}^{+}+s^{4} \alpha_{4}^{-}+s^{3} \alpha_{3}^{-}+s^{2} \alpha_{2}^{+}+s \alpha_{1}^{+}+\alpha_{0}^{-}$

$P_{o p}^{++}=s^{6}+s^{5} \alpha_{5}^{+}+s^{4} \alpha_{4}^{+}+s^{3} \alpha_{3}^{-}+s^{2} \alpha_{2}^{-}+s \alpha_{1}^{+}+\alpha_{0}^{+}$

$P_{o p}^{+-}=s^{6}+s^{5} \alpha_{5}^{-}+s^{4} \alpha_{4}^{+}+s^{3} \alpha_{3}^{+}+s^{2} \alpha_{2}^{-}+s \alpha_{1}^{-}+\alpha_{0}^{+}$

A Routh Tabulation with 6 free multi-linear coefficients, can only be solved for the stabilizing interval of a variable, if and only if, other 5 parameters were fixed. Therefore a new algorithm as an iterative method is introduced in Fig. 2. 


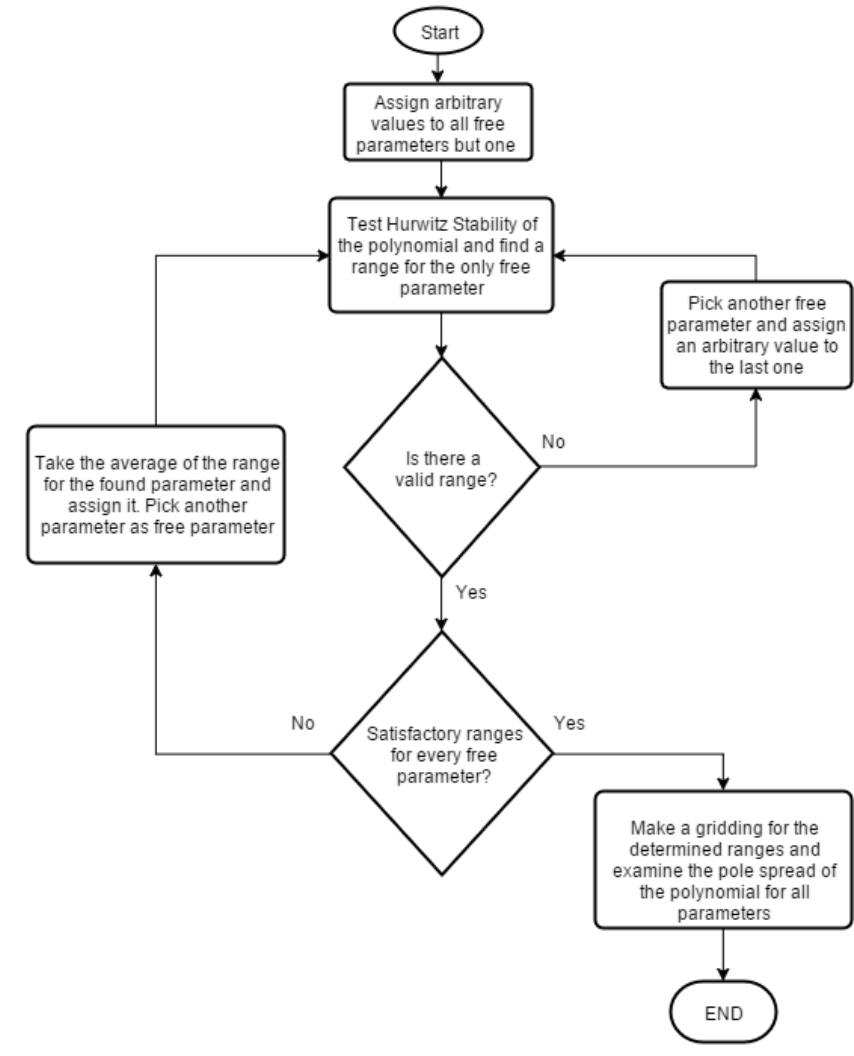

Fig. 2. Flowchart of the algorithm: The iterative method that is used to determine the range of the stabilizing feedback.

Four Kharitonov Polynomials are examined iteratively for smaller intervals of " $\mathrm{k}$ " until a stable overbounding polynomial was obtained (16) according to the algorithm introduced above.

The region obtained by the overbounding polynomial has a narrow range for " $k$ " values. This is natural since the overbounding polynomial approach is conservative. Pole spread is the pole map of a polynomial family in Laplace domain, for each possible value that the free parameters can take [8]. The stable region for all " $k$ " values and the pole spread of the characteristic polynomial in this region is given in Fig. 3.

$0.0012>k_{1,2,3,4,5,6}>0.0013$

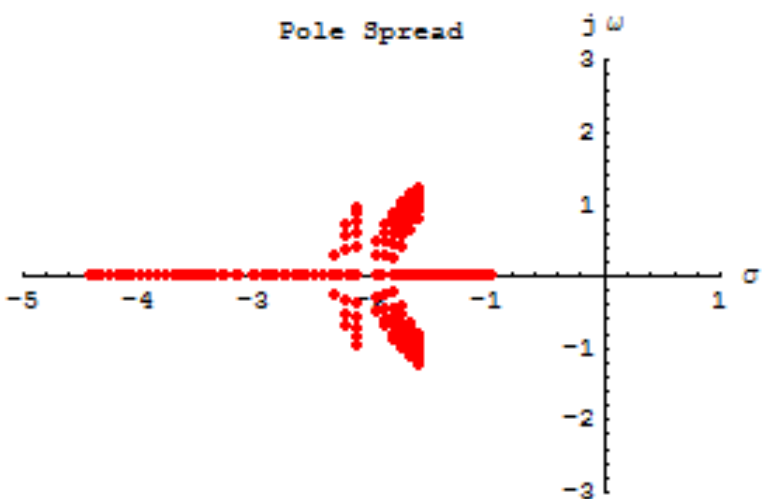

Fig. 3. Pole spread: Poles of the closed-loop system for all stabilizing feedback gain values.
It is possible to check for stability beyond the determined range of " $k$ " values that have been found. It is possible to see that this range that has been found is a conservative one and can be extended beyond. Moreover, gamma-stability and pole placement for the desired response was not considered, only the Hurwitz stability of the system was examined. Therefore, this is a very conservative solution and considers just one interval.

\section{B. Robust Partial Pole Assignment Approach}

As we obtained the closed-loop characteristic equation of the system, now we need to determine the variables in the equation. As shown in Table 1 " $b$ " values regarding the structure of the system are given as constants. However, 6 variables for 6 " $\mathrm{k}$ " values exist. As these "k" values would affect the dynamics and the stability of the system, it is important to design the " $\mathrm{K}$ " matrix as robust as possible.

Robust partial pole assignment secures the location of the desired poles while trying to keep the other poles in a specified region [9]. This region is usually referred to as "Gamma Region" also introducing the term "Gamma Stability".

Robust partial pole assignment consists of a few steps and iteration to determine all the stabilizing feedback gain coefficients in a similar way to "Pole Retention" [10]. This way, both the stability and a good response is maintained while designing the state feedback. Desired Pole Locations can be defined using (17):

$$
P(s)_{\text {desired }}=\left(s^{2}+2 \zeta \omega_{n} s+\omega_{n}^{2}\right)+(s+p)
$$

This equation assigns 3 three poles to their desired locations while leaving other poles free. The notion that has been introduced Gamma-Stability will be applied to keep the other poles away from the dominant area while giving a range of " $k$ " values that satisfy the stability condition. Since the "A" matrix is a $6 \times 6$ matrix, 3 poles still remain free and can be expressed using a residue polynomial:

$$
P(s)_{\text {desired }}=s^{3}+s^{2} a_{2}+s a_{1}+a_{0}
$$

Multiplication of these two polynomials can be designed to comply with the closed-loop polynomial that is found earlier by tuning the " $\mathrm{k}$ " values concerning the coefficients of the residue equation.

If we assume that the residue polynomial is gammastable and the desired polynomial is determined, multiplication of these two polynomials should be achievable by the state feedback design parameters. According to [11] that if the degree of freedom, namely number of free parameters are greater than the poles to be assigned, the solution is non-unique and a set of solutions for feedback gain values can be obtained.

The closed-loop characteristic polynomial in terms of " $k$ " values is equalized to the polynomial of the desired and residue poles which will be denoted by $\mathrm{Pc}(\mathrm{s})$ for the same powers of "s" to obtain a set of equations. 
$P_{c}(s)=P(s)_{\text {desired }} P(s)_{\text {residue }}$

$P_{c}(s)=s^{6}+s^{5}\left(p+\alpha_{2}+2 \zeta \omega_{n}\right)+$

$s^{4}\left(a_{1}+p a_{2}+\omega_{n}^{2}+2 p \zeta \omega_{n}+2 a_{2} \zeta \omega_{n}\right)+$

$s^{3}\left(p a_{1}+a_{0}+p \omega_{n}^{2}+\alpha_{2} \omega_{n}^{2}+2 a_{1} \zeta \omega_{n}+2 p a_{2} \zeta \omega_{n}\right)$

$+s^{2}\left(p a_{0}+a_{1} \omega_{n}^{2}+p \alpha_{2} \omega_{n}^{2}+2 p a_{1} \zeta \omega_{n}+2 a_{0} \zeta \omega_{n}\right)$

$+s\left(p \alpha_{1} \omega_{n}^{2}+\alpha_{0} \omega_{n}^{2}+2 p a_{0} \zeta \omega_{n}\right)+p a_{0} \omega_{n}^{2}$.

Equating $\mathrm{Pc}(\mathrm{s})$ to $\mathrm{P}(\mathrm{s}) \mathrm{ch}$ yields 6 linear equations with 9 free parameters since we assume that the coefficients of the desired polynomial equation are known. It is easy to assign poles to desired locations but we shall pursue a range for " $k$ " values to make the residue polynomial gamma-stable in the given range. This problem will be solved in the case study section in three parts. The first part introduces " $k$ " values, and the second part is about the performance of the designed feedback gains. Robustness and robust performance are examined in part three.

\section{CASE Study}

\section{A. Determination of All Stabilizing $K$ Values Regarding Gamma Stability}

This section presents numerical examples and results of the proposed method. The feedback design problem is considered with the numerical values in Table 1. Moreover, design criteria are specified for the assigned poles and the first row of the feedback will be defined with constant values in order to apply the pole retention method regarding other 4 free " $\mathrm{k}$ " parameters.

First of all, for an acceptable desired response two poles will be assigned at $[-2+2 \mathrm{i},-2-2 \mathrm{i}]$ and the third pole will be assigned to [-3]. A gamma stability region of " $\mathrm{s}=-3$ " line will be considered and all the remaining poles, namely residue poles will be placed to the left of the "s=-3" line. Arbitrary values for $\mathrm{k} 1=0.005$ and $\mathrm{k} 2=0.003$ will assigned to reduce the problem to a narrower context. However, this was not necessary since we had more degrees of freedom than the residue poles. A range for " $\mathrm{k} 3,4,5,6$ " will be determined for angle stabilization. For the "b" values in Table 1 and the coefficients for the design criterion, the following equations can be obtained.

$$
\zeta=0.707, \omega_{n}=2.838, p=3, k_{1}=0.005, k_{2}=0.003
$$

$$
\begin{aligned}
& a_{0}=6.7^{6} k_{3} k_{5} \\
& a_{1}=-0.0417\left(3.753^{7} k_{3} k_{5}-1.608^{8} k_{4} k_{5}-1.608^{8} k_{3} k_{6}\right) \\
& a_{2}=-0.0417\left(-41905 k_{3}-55555 k_{5}+3.58 k_{3} k_{5}-\right. \\
& 9.65^{7} k_{4} k_{5}-9.65^{7} k_{3} k_{6}-1.608^{8} k_{4} k_{6}-0.833 \\
& \left.\left(3.75^{7} k_{3} k_{5}-1.608^{8} k_{4} k_{5}-1.608^{8} k_{3} k_{6}\right)\right) .
\end{aligned}
$$

These three equations are substituted into the residue polynomial since we have already assigned the other three pole and here we will introduce the gamma-stability into the equation. The "s" variable in the equation will be substituted by " $s+3$ " and the new polynomials Hurwitz stability will be enough to make sure that the gamma-stability condition is satisfied.

A conservative solution and the pole spread of all stabilizing " $k 3,4,5,6$ " values (22) is shown in Fig. 4

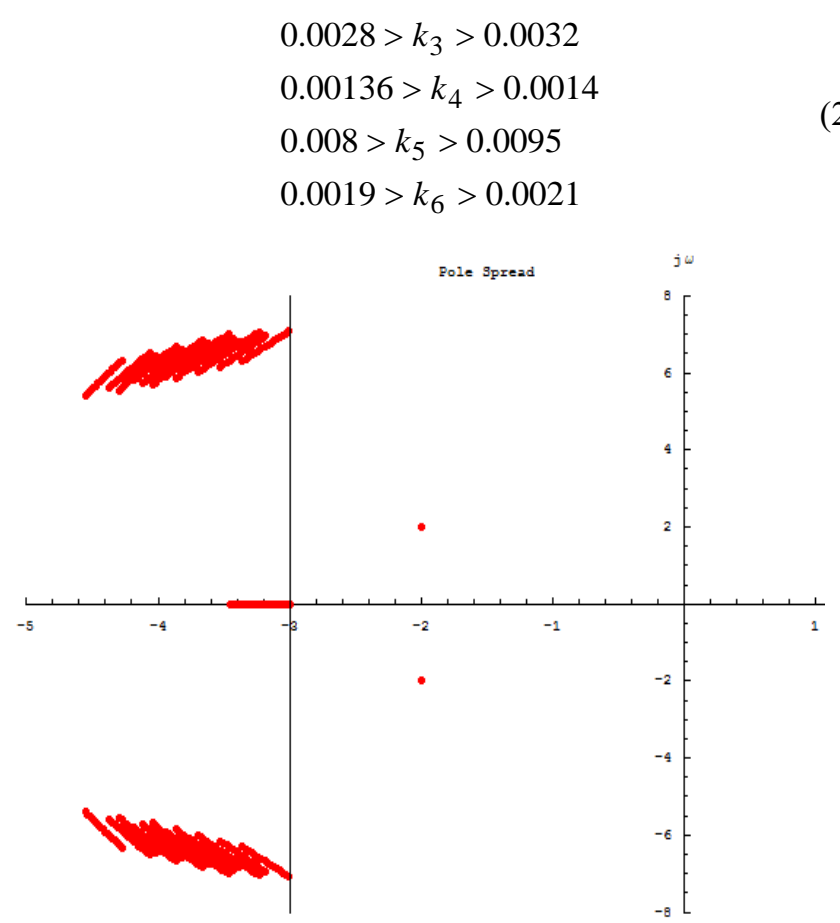

Fig. 4. Pole spread: Poles of the closed-loop system for all stabilizing feedback " $k 3,4,5,6$ " values.

It can be seen that the pole spread ensures the locations of the assigned poles while keeping all other poles in the nondominant area, which demonstrates that the gamma-stability criterion is satisfied.

It is worth mentioning that since the polynomial has multi-linear coefficients and the solution to the desired pole locations is not unique in Multi-Input Multi-Output systems [12], there are numerous ranges for each " $\mathrm{k}$ " value regarding other " $k$ " ranges. The range of " $k$ " values that we have found, using the numerical iterative approach with Routh tables, is a stabilizing region for the fixed values of " $\mathrm{k} 1$ " and "k2".

\section{B. Performance Analysis of the Assigned Poles}

In this part, performance analysis is made for assigned poles to see the response of the system. Since the control problem is the angle stabilization, the most convenient way to test it is to start the system with zero reference signal and with initial conditions such as unbalanced angles. The initial condition response of the system for the following angles (Roll Angle $=0.3 \mathrm{rad}$, Pitch Angle $=-0.2 \mathrm{rad}$, Yaw Angle $=0.1$ $\mathrm{rad})$ is shown in Fig. 5. It can be seen that a satisfactory initial condition response is achieved with the designed state feedback shown in (23):

$$
K=\left|\begin{array}{cccccc}
0.005 & 0.003 & 0 & 0 & 0 & 0 \\
0 & 0 & 0.003 & 0.0014 & 0 & 0 \\
0 & 0 & 0 & 0 & 0.009 & 0.002
\end{array}\right|
$$




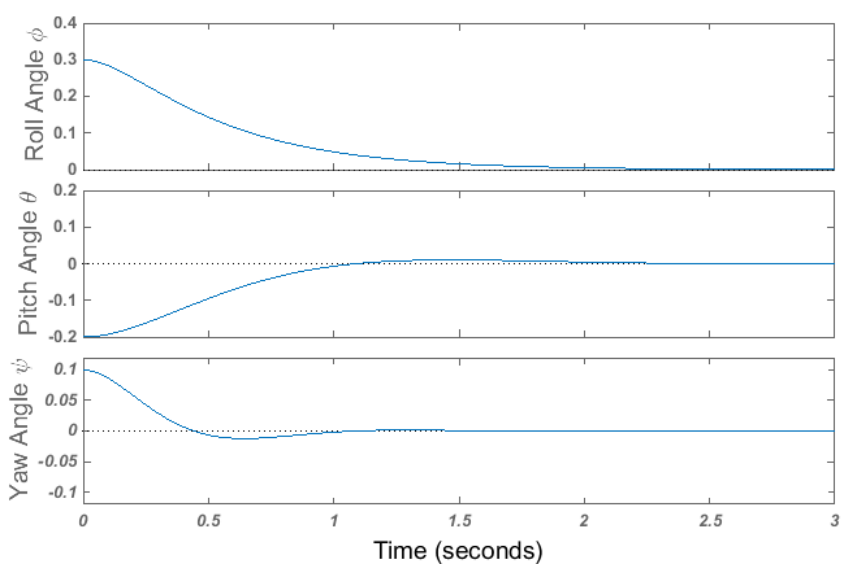

Fig. 5. Response to initial conditions with the designed state feedback for the initial state values $(\phi=0.3 \mathrm{rad}, \theta=-0.2 \mathrm{rad}, \psi=0.1 \mathrm{rad})$

\section{Robustness and Performance Analysis}

In this section, the proposed design is examined by taking uncertain parameters in the " $\mathrm{A}$ " matrix into account. These parameters were previously neglected as gyro effects. The new "A" matrix regarding the uncertain perturbations including the gyroscopic effects is shown in (24) with the bounds on the uncertain parameters (25)

$$
\begin{gathered}
A(q)=\left|\begin{array}{cccccc}
0 & 1 & 0 & 0 & 0 & 0 \\
0 & 0 & 0 & 0 & q_{1} & 0 \\
0 & 0 & 0 & 1 & 0 & 0 \\
0 & q_{2} & 0 & 0 & 0 & 0 \\
0 & 0 & 0 & 0 & 0 & 1 \\
0 & 0 & 0 & 0 & 0 & 0
\end{array}\right| \\
-0.6<q_{i}<0.6 \rightarrow i=1,2
\end{gathered}
$$

Pole spread of the uncertain closed characteristic polynomial obtained for the uncertain $\mathrm{A}(\mathrm{q})$ matrix in (24), is shown in Fig. 6. Gamma-Stability region border is shown with a vertical green line, $\zeta=0.707$ lines are represented by $45 \mathrm{o}$ green lines from the origin and the desired pole locations are circled with blue circles to see if the desired pole locations were allocated by the perturbations caused by uncertain gyro effects.

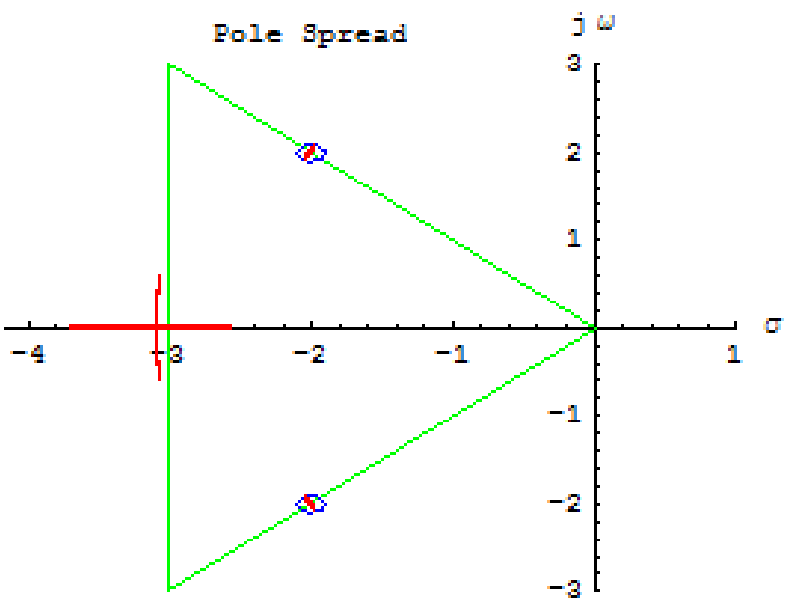

Fig. 6. Pole spread: Poles of the closed-loop system for all possible A(q) matrices in red.
The pole spread of all possible polynomials of $A(q)$ matrix according to the uncertain parameter " $q 1,2$ " is plotted by gridding the "q" values from -0.6 to 0.6 with small step sizes such as 0.01 as shown in (25). The resulting pole spread has the desired characteristics for all combinations of "q". It can be seen that the poles placed at $[-2+2 i,-2-2 i]$ stay in a circle of radius 0.1 , so their characteristics are maintained. The gyro effects do not affect the yaw angle, therefore only the response to initial conditions for the roll and the pitch angles are simulated in Fig. 7.

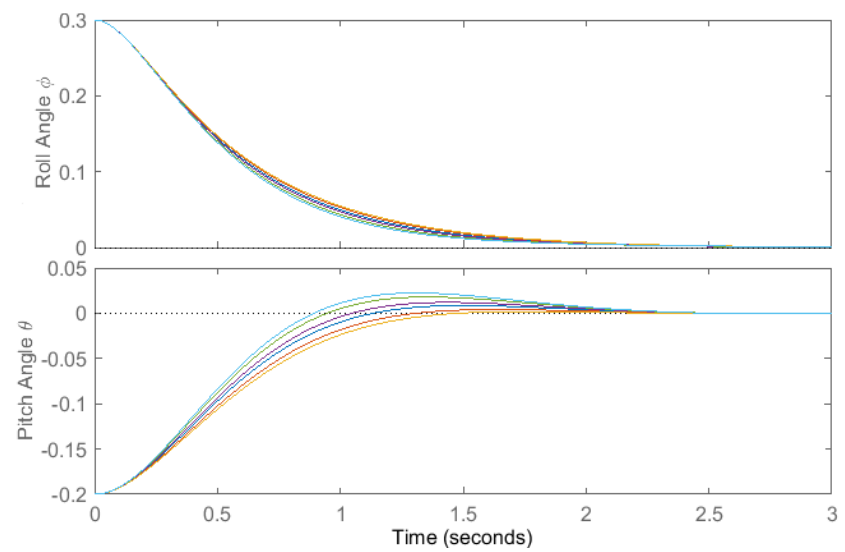

Fig. 7. Response to initial conditions: Each initial condition $(\phi=0.3 \mathrm{rad}, \theta=$ $-0.2 \mathrm{rad}$ ) response of the uncertain system $\mathrm{A}(\mathrm{q})$, where " $\mathrm{q}$ " varies as in (25), is plotted with a different color. All the colored signals are stable, hence the design is robustly stable.

Since the proposed method is a robust pole assignment method, perturbations in the "A" matrix are almost neutralized as it can be seen in Fig. 7. The uncertain system (24) is stable for all values of "q" bounded as in (25) with the designed state feedback (23).

\section{CONCLUSION}

A new numerical iterative method to find the stabilizing feedback gain values were found, for a set of design criterion and gamma-stability. Results are simulated on a quadrotor, which can be defined as an uncertain MIMO system if the gyro effects are also considered. Robust design methods are useful when parametric uncertainties are present in the linear time-invariant system. Usually, robust control approaches lead to conservative solutions but we have used a pole assignment method to get satisfactory performance without compromising the stability.

The methods introduced in this paper can be applied to different linear time-invariant systems as well. The application of robust design methods guarantees the stability of a system for a range of determined state feedback values. Starting with the Kharitonov Polynomial Approach, a conservative solution is found. Then the dominant poles are placed using the pole retention method and the design is expanded while the stability is guaranteed. As it is seen in the case study, the designed state feedback stabilizes the system for a variety of "A" matrices.

\section{REFERENCES}

[1] Villbrandt, J. (2010, July 1), "The Quadrotor's Coming of Age", Aerospace Engineering, Issue II, Security \& Defense, Volume XII, Retrieved from URL: http://illumin.usc.edu/162/the-quadrotorscoming-of-age. 
[2] Raza, A. S., and Gueaieb, W. (2010). Intelligent Flight Control of an Autonomous Quadrotor: In Motion Control, pp. 245-264., IN-TECH, ISBN: 978-953-7619-55-8.

[3] Bouabdallah, S., Murrieri, P., and Siegwart, R. (2004), "Design and control of an indoor micro quadrotor," IEEE International Conference on Robotics and Automation, 2004. Proceedings. ICRA '04. 2004, New Orleans, LA, USA, 2004, pp. 4393-4398 Vol.5. doi: 10.1109/ROBOT.2004.1302409

[4] Bouabdallah, S. (2007). Design and Control of quadrotors with application to autonomous flying. 10.5075/epfl-thesis-3727.

[5] Ackermann J., Hu H. Z., and Kaesbauer D. (1988), "Robustness analysis: a case study," Proceedings of the 27th IEEE Conference on Decision and Control, Austin, TX, USA, 1988, pp. 86-91 vol.1. doi: 10.1109/CDC.1988.194274

[6] Ackermann, J. (2002), Robust Control: The parameter space approach, Springer, London, ISBN: 1-85233-514-9.

[7] Bhattacharyya, S. P., \& Keel, L. H. (1995). Robust control: the parametric approach. In Advances in Control Education 1994 (pp. 4952). Pergamon
[8] Soylemez, M. T. (1999), Pole Assignment for Uncertain Systems, Research Studies Press, Baldock, UK, ISBN: 0-863-80246-X

[9] Soylemez, M. T., \& Munro, N. (2001). A parametric solution to the pole assignment problem using dynamic output-feedback. IEEE Transactions on Automatic Control, 46(5), 711-723.

[10] Zheng, X., Zolotas, A. C., \& Wang, H. (2006). Mathematica implementation of output-feedback pole assignment for uncertain systems via symbolic algebra. International Journal of Control, 79(11), 1431-1446.

[11] Soylemez, M. T., \& Munro, N. (1998). A new technique for partial pole placement using constant output-feedback. In Proceedings of the 37th IEEE Conference on Decision and Control (Cat. No. 98CH36171) (Vol. 2, pp. 1722-1727). IEEE.

[12] Munro, N., Kontogiannis E., and Nuttall B. (1994), "Robust control system analysis and synthesis." In IEE CONFERENCE PUBLICATION, pp. 583-583. INSTITUTION OF ELECTRICAL ENGINEERS, 